\title{
EXPLOSIÓN DEMOGRÁFICA \\ Y TENSIÓN ECONÓMICA
}

\author{
E. R. Weiss-Altaner * \\ Universidad de Pennsylvania
}

\section{INTRODUCCIÓN}

EN LOS ULtimos años ha aumentado la preocupación, difundida desde los Estados Unidos hasta otras parte del mundo, por el crecimiento de la población. Esta inquietud proviene del temor de que la población, tanto a escala nacional como mundial, se esté acercando rápidamente a los límites del crecimiento y con ello a un desenlace catastrófico. Tal preocupación ha revestido en ocasiones un carácter histérico. ${ }^{1}$ El problema se manifiesta especialmente amenazador en los países menos desarrollados. Se afirma que el constante crecimiento demográfico representa una seria amenaza, a veces la más grave, para la calidad de la vida y para la posibilidad misma de que perdure la especie humana. La reconsideración más en boga ha sido la de reducir la fecundidad. Con este propósito algunas instituciones privadas y gubernamentales de los Estados Unidos han financiado propaganda y programas de índole práctica encaminados a lograr que el público adquiera conciencia sobre "la crisis demográfica" y las ventajas del descenso de la fecundidad. La mayoría de los países de América Latina cuentan ya con alguna experiencia en programas de población patrocinados por organismos estadounidenses.

Un nuevo planteamiento en esta materia ha sido el rechazo absoluto del crecimiento continuado, no sólo de la población sino también de la producción. La formulación más vigorosa y más reciente de este enfoque figura en el ensayo citado, elaborado para el Club de Roma, The Limits to Growth.

Estas obras y otras de la misma índole afirman que la población está sobrepasando rápidamente la capacidad del planeta para sostenerla, previendo la destrucción por efecto de hambrunas, pestes y en un futuro muy cercano, la guerra, a menos que la población deje de crecer o dis-

* El autor agradece los comentarios de José B. Morelos, del Centro de Estudios Económicos y Demográficos, El Colegio de México, referentes a una versión anterior de este ensayo.

1 Paul Ehrlich, The Population Bomb, Nueva York, Ballantine Books, 1968. Otros ensayos sobre este problema que han tenido gran publicidad en Estados Unidos son: Paul y Anne Ehrlich, Population, Resaurces, Environment, San Francisco, W. H. Freeman, 1970; Commission on Population Growth and the American Future, Population and the American Future, Washington, D. C., 1972; D. H. Meadows, D. L. Meadows, I. Randers, W. W. Behrens III, The Limits to Growth: a Report for the Club of Rome's Project on the Predicament of Mankind, Nueva York, Universe Books, 1972; versión en español, Los limites del crecimiento, México, Fondo de Cultura Económica, 1972. 
minuya su ritmo de crecimiento. Augura que aun cuando se restrinja el crecimiento demográfico, el crecimiento de la producción seguirá sometiendo a una explotación excesiva los limitados recursos del planeta, amenazando con agotarlos, también en un futuro no lejano; no sólo se extinguirán los recursos, sino que la tierra se arruinará como resultado de la contaminación del medio, inherente a los métodos más avanzados de producción.

Dado que lo anterior constituye solamente una interpretación, entre varias, del complejo población-economía, cabe preguntarse cuáles son algunos de los hechos no discutidos. La población crece rápidamente, sobre todo en los países de menor desarrollo relativo y al mismo tiempo el uso de los recursos y la contaminación crecen aceleradamente, al paso que la desigualdad económica y el conflicto social perduran, si no es que aumentan. Ciertamente, el tamaño y el crecimiento de la población están relacionados con el empleo de los recursos, la contaminación y el conflicto, pero ¿en qué forma ocurre esto? ¿cuáles son los vínculos precisos entre los cambios demográficos y las transformaciones económico-ambientales, y cuál es la importancia relativa de los diversos nexos? Por último, ¿qué importancia reviste un descenso de la fecundidad en esta concatenación de problemas sociales y económicos?

La idea principal en este ensayo es que el crecimiento demográfico no constituye la causa más importante de los problemas sociales y económicos con que se enfrentan actualmente los países y que la disminución de la fecundidad no es la solución más importante para esos problemas. Parecen mucho más decisivos los factores estructurales tales como la distribución del ingreso y la distribución del poder, o las relaciones sociales de producción. En realidad, en esta fase de nuestra historia, las tensiones que afloran en un período de crecimiento demográfico rápido, pueden ser más un síntoma de la incapacidad de las actuales relaciones sociales de producción para satisfacer adecuadamente las nuevas demandas, que un indicio de que la población se aproxima al número máximo que un país o que el mundo puede llegar a sustentar.

Aun teniendo en cuenta las proyecciones más favorables en materia de innovación tecnológica y de las relaciones sociales, es posible imaginar una situación futura en que la población haya alcanzado los límites para su expansión, pero ¿cuándo se dará esta circunstancia? ¿pronto o muy tarde? ¿en un futuro demasiado distante como para que dediquemos exclusivamente nuestra atención al tamaño de la población mientras se olvidan las desigualdades estructurales y otros obstáculos de indole estructural? Parece que entre los años 70 y 80 , al igual que en el pasado, estas últimas condiciones representan los obstáculos más importantes.

El análisis que sigue gira en torno a cuatro asociaciones generales en las que interviene la población:

1) Población-producción

2) Población-contaminación

3) Población-urbanización

4) Población-política 


\section{PoBlación Y PRODUCCión ${ }^{2}$}

Los problemas demográficos no se reducen exclusivamente a la tasa de crecimiento de la población o al tamaño absoluto de una población. Desde un punto de vista económico, el crecimiento demográfico genera demandas de empleo y de consumo, las cuales, de no ser satisfechos dan lugar al surgimiento de la tensión económica. ${ }^{3}$ Denominamos "tensión económica" al efecto resultante de la interrelación entre las variables demográficas por una parte, y las variables económicas, por la otra. El crecimiento demográfico genera demandas adicionales que exigen un incremento de la oferta. De esta manera, la tensión económica resulta de una situación de desequilibrio entre la demanda y la oferta. Las principales consecuencias del desequilibrio entre oferta y demanda que generan tensión económica son:

a) Problemas de consumo: Un desequilibrio entre las necesidades de consumo (demanda de bienes y servicios) y la producción (oferta de los mismos) y;

b) Problemas de empleo: Un desequilibrio entre la oferta de fuerza de trabajo y las posibilidades de empleo (demanda de fuerza de trabajo).

En este sentido, un cambio en el grado de tensión económica puede resultar de un aumento de la población o de un cambio en las condiciones económicas o de la combinación de ambos. Así, un descenso súbito en la demanda de productos de una región puede generar un exceso de oferta de fuerza de trabajo aun sin que se altere el volumen de dicha fuerza. Sin embargo, interesan mayormente los desequilibrios creados inicialmente por un rápido crecimiento demográfico y sus efectos posteriores.

En sentido económico "la crisis demográfica" es de índole diversa: la tensión económica es el resultado de un desequilibrio entre factores

2 El profesor Richard A. Easterlin de la Universidad de Pennsylvania ha tenido gran influencia en la determinación del enfoque adoptado en este trabajo, aunque naturalmente las opiniones presentadas aquí son de la exlusiva responsabilidad del autor. Véanse las obras de Easterlin: "Effects of Population Growth on the Economic Development of Developing Countries", Annals of the America Academy of Political and Social Science, Vol. 369, enero de 1967, pp. 98-108; "Relations between Population Pressure and Economic and Demographic Change", Conferencia Internacional de Población, Londres, Unión Internacional para el Estudio Científico de la Población, Vol. III, 1969, pp. 1661-1674.

3 Posteriormente a la elaboración de este ensayo, el autor conoció la obra del economistas húngaros János Kornai, Anti-Equilibrium, Amsterdam, North Holland Publishing Company, 1971, en la cual Kornai usa un concepto de tensión semejante a "la tensión económica". Este concepto de Kornai es un elemento clave en su crítica del modelo de equilibrio general que actualmente impera en el pensamiento económico burgués o neoclásico. Kornai esboza un lenguaje teórico para analizar el desequilibrio permanente, que según él constituye la situación distintiva de la economía y no precisamente el equilibrio.

${ }_{4}$ Aun cuando el concepto de tensión no se restringe al desequilibrio entre la demanda y oferta económicas, estrictamente hablando, también es cierto que el crecimiento demográfico por sí solo no basta para producir una situación de tensión, son indispensables tanto un conjunto de demanda como otro de oferta. 
múltiples que pueden agruparse para un problema dado, según sus efectos sobre la demanda o sobre la oferta. La fecundidad es uno de los factores clave que contribuyen a la tensión económica aunque tal vez no el más importante. En este sentido cabría preguntarse si es válido imputar el desempleo a la fecundidad sólo porque influye directamente en el volumen de la fuerza de trabajo. Si en otro sentido se considera que el desempleo es el resultado de las condiciones de oferta y demanda, podría igualmente imputarse a cambios en la demanda de fuerza de trabajo.

Gran parte de la controversia acerca de la relación entre población y producción gira alrededor de la siguiente pregunta: ¿Es posible que la producción aumente ante la presencia de un rápido aumento demográfico de modo que se sostenga y mejore el ingreso per capita?

El análisis de las relaciones entre esta diversidad de variables que intervienen en el proceso demográfico es posible realizarlo a través de una función producción ${ }^{5}$ en la que se incluyan los posibles factores que constituyen, en apariencia, las fuentes de los cambios en la producción:

$$
Q=f\left(K, K^{\prime}, L, L^{\prime}, T, A, D\right)
$$

en donde:

$Q=$ producción

$K=$ bienes de capital

$K^{\prime}=$ calidad de los bienes de capital

$L=$ fuerza de trabajo

$L^{\prime}=$ calidad de la fuerza de trabajo

$T=$ recursos naturales

$A=$ asignación de recursos

$D=$ efectos de escala

El cambio tecnológico está incorporado implícitamente dada su influencia en la producción, principalmente a través de $K^{\prime}, L^{\prime}$ y $D$. La influencia combinada de otros aspectos del cambio tecnológico y de otros insumos directos no precisados, se refleja en el desplazamiento de la función $f$ en el tiempo.

Además de estos factores directos existen variables, que llamaremos "los factores indirectos de producción", que influyen en el nivel de la producción a través de los insumos directos. Entre ellas se encuentran: la distribución del ingreso, la tenencia de la tierra, la educación, la salud, la vivienda, la administración pública, etc. Todos estos factores están relacionados entre sí y algunos forman parte de lo que en el vocabulario marxista se conoce como "las relaciones sociales de producción". El análisis de los cambios de estas variables y de sus efectos, podría ser de gran significación en el diseño de políticas que ayuden a resolver situaciones de tensión económica. La importancia relativa de los diversos

5 El concepto de función producción se usa aquí en el sentido de una relación técnica o de ingeniería, y principalmente para distinguir los insumos directos de los factores indirectos en la producción. El autor no rechaza la crítica devastadora hecha por la profesora Joan Robinson de la Universidad de Cambridge y por otros autores sobre la función agregada de producción neoclásica. 
factores indirectos, entre sí y con respecto a la fecundidad, podría demostrarse mediante estudios históricos de casos concretos.

Cabe observar que los factores indirectos de producción constituyen el marco dentro del cual se desenvuelven los insumos directos. Los factores indirectos de producción son parámetros: limitan en cierta forma el funcionamiento de los insumos directos. Por ejemplo, una justificación de la reforma agraria en la América Latina reside en que la gran concentración de la propiedad de las tierras ha limitado el crecimiento de la producción y del empleo en la agricultura. Para lograr una oferta adecuada de alimentos por habitante, ha sido necesario luchar por una reforma institucional - por un nuevo régimen de tenencia de tierrasa fin de brindar a los insumos directos libertad para elevar la producción agrícola.

En este contexto se analizan dos modelos que han tenido gran difusión:

El modelo malthusiano clásico cuya expresión algebraica es:

$$
Q=f(L, T)
$$

en el cual se supone que los insumos se combinan en proporciones fijas, que $T$ (los recursos naturales) no varía, y que no hay cambios tẹcnológicos.

En este modelo, a medida que la población crece, otro tanto ocurre con la fuerza de trabajo. Sin embargo, a causa de los rendimientos decrecientes de la fuerza de trabajo (todos los demás insumos son constantes), la producción aumenta menos que proporcionalmente con respecto a la población, y el producto por habitante disminuye.

El hecho de que se suponga constante la técnica (proporciones fijas de insumos) y de que se descarten las innovaciones, restringe la utilidad de este modelo, particularmente en nuestra época, en la que una proporción predominante del crecimiento secular de la producción desde 1750 se atribuye a las mejoras de la tecnología.

El otro modelo constituye una formulación más reciente del anterior y supone que los incrementos en la inversión neta y en los bienes de capital y su calidad, son los determinantes principales de los aumentos de la producción:

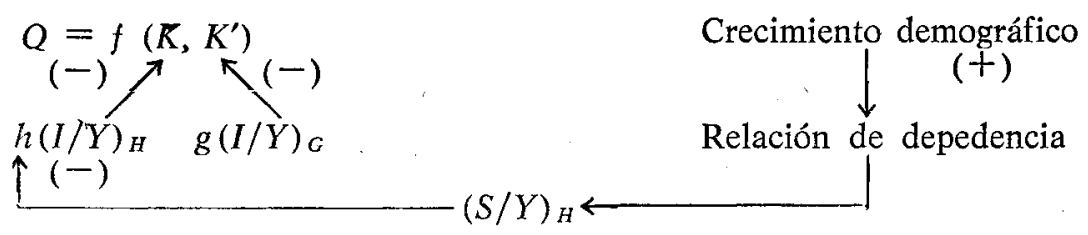


En este modelo el crecimiento demográfico ejercería sus efectos a través de la proporción del ingreso que se invierte, $I / Y$, la cual se divide en inversión de los hogares e inversión del gobierno:

$$
I / Y=(I / Y)_{H}+(I / Y)_{G}
$$

Según el modelo, a medida que la población aumenta $(I / Y)_{H}$, la proporción del ahorro (ex post) en el ingreso de los hogares disminuye. A su vez, el crecimiento demográfico hace que aumente la proporción de personas dependientes, o relación de dependencia, haciendo necesaria una redistribución del ingreso del hogar encaminada a dedicar una proporción mayor para fines de consumo. Esto reduce la contribución del ahorro de los hogares a los fondos disponibles para inversión. Mientras tanto, a escala nacional, el incremento de la proporción de dependientes conduce a una redistribución de la inversión pública hacia vivienda, escuelas, y salud pública, o sea hacia inversiones menos productivas.

Este argumento puede ser objeto de críticas. Paul Israel Singer ${ }^{6}$ y Angel Fucaraccio insisten en que debe incorporarse el factor (indirecto) distribución del ingreso. Advierten que las clases sociales que registran fecundidad más elevada son las de ingresos más bajos. Consideran que los cambios en la fecundidad tendrían escaso efecto en la proporción del ingreso que se destina al ahorro, dado que todo o casi todo se seguiría dedicando, en todo caso, a fines de consumo.

Además, sería necesario considerar hasta qué punto es cierto que la inversión en vivienda y educación es considerablemente menos productiva que la inversión en fábricas y equipo productivo. Las mejorías en los niveles de enseñanza y condiciones de la vivienda, por lo menos en lo que se refieren a la calidad de la fuerza de trabajo, pueden influir positivamente sobre la producción. ${ }^{7}$ La validez de estas cuestiones, para determinados países y épocas, queda aún por estudiarse. No obstante, dado que los gastos en educación y vivienda alcanzan niveles muy reducidos, cabe esperar que su elevación hará crecer la producción en forma proporcional o más que proporcional, caeteris paribus. Además es muy probable que dados los bajos niveles de gasto por persona en educación y vivienda, se generen rendimientos marginales crecientes con la aplicación de los mismos.

Por otra parte, es necesario considerar que el modelo no contempla la posibilidad de innovación. Este factor juega un papel de primera importancia en la enseñanza en virtud de aue la tarea de educar a una población en crecimiento, exige la multiplicación correlativa de los servicios educacionales. En este sentido, por ejemplo, la duplicación de la población implicaría duplicar los insumos destinados a la educación a fin de elevar al doble el producto de la enseñanza y mantener inalterado el nivel de educación por persona. Esto significaría que, a medida que la población crece, la sociedad tendría que gastar una proporción cada vez

- Paul Israel Singer "Cambios de población y producción"; Angel Fucaraccio, "El Control de la natalidad y el argumento del ahorro y la inversión", en Actas de la Conferencia Regional Latinoamericana de Población. México, 1970, El Colegio de México, Vol. II, 1972, pp. 51-56 y 57-63 respectivamente.

7 E. H. Phelps-Brown, The Economics of Labor, New Haven, Yale University Press, 1962, Cap. 3. Véase también la abundante literatura sobre "capital humano". 
mayor de sus recursos de inversión para evitar el deterioro de las condiciones educativas.

Es claro que existe la posibilidad de cambiar las proporciones de insumos, de modificar la calidad de los mismos y de transformar la estructura en que se desenvuelve la producción. Si nos atenemos a la crítica de Ivan Illich del Centro Interamericano de Documentación (CIDOc), cuando afirman que la escuela constituye el principal obstáculo para la enseñanza, tal vez la presión de la población constituya suficiente incentivo para justificar otras formas de impartir la enseñanza. Esas innovaciones podrían permitir que el costo de un grado determinado de enseñanza permanezca relativamente estable y que al mismo tiempo su calidad aumente.

Estos modelos han sido criticados por su estrecha concepción sobre las fuentes del crecimiento de la producción, particularmente por la exclusión de las innovaciones tecnológicas. Sin embargo, existe una corriente nueva, representada por los científicos que prepararon el informe para el Club de Roma, en la que se reconoce la importancia del cambio tecnológico aún cuando sostiene que los recursos naturales están casi agotados y que las innovaciones no pueden aumentar con la suficiente rapidez como para evitar el desastre. De cualquier modo, al final el tamaño de la población se considera que debe estabilizarse. Sin embargo, no está demostrado que el límite máximo de explotación de los recursos naturales haya sido alcanzado. A este respecto, la Academia Nacional de Ciencias de los Estados Unidos informó que sólo un poco más de la mitad de las tierras mundiales, potencialmente cultivables (que estimaban en 1450 millones de hectáreas) se encontraban en proceso de explotación, o sea que está aún distante el límite máximo de explotación de las tierras agrícolas. Ante esto quedaría entonces por averiguar si la superficie sin utilizar puede someterse a explotación y si es posible hacerlo con la tecnología existente. Y, por otra parte. si la inelasticidad de la oferta de tierras agrícolas no se debe primordialmente a un agotamiento de la existencia de las mismas, cabe preguntarse cuál es la o las causas de tal inelasticidad $\mathrm{y}$, análogamente, cómo se explica la inelasticidad de la oferta de los demás factores de producción y en especial la de la tecnología.

Tal vez la respuesta a estas interrogantes se encuentre en el comportamiento de los factores indirectos de la producción. La distribución del ingreso y la estructura social a que da lugar, suelen presentarse como manifestaciones de los problemas demográficos, cuando en realidad son los que en cierta medida los generan. La tensión económica no se manifiesta de manera uniforme dentro de la mayor parte de las sociedades, pues sabemos que existen diferencias de clases, apreciables a través de las variables que la producen. Cualquier política encaminada a superar la tensión, debe tener presente los efectos distributivos. A manera de ejemplo, podríamos imaginar un caso en el que una disminución de la fecundidad registrada en las clases más pobres y con tasa elevada de crecimiento, se tradujera en una disminución de la tensión económica nacional (meramente un cambio aritmético) sin ninguna alteración en la

8 John S. Chapman, "Food from the Land", Resources and Man, San Francisco, W. H. Freeman, 1969, p. 67. 
tensión económica que viven esas clases. En efecto, podría haber ocurrido que la desigualdad de ingreso no cambiara e incluso que empeorara. Además, la fecundidad no está relacionada estrechamente con la elasticidad de la oferta de los factores directos de producción, mientras que los factores indirectos sí lo están.

Otra característica de gran parte de los trabajos sobre "la crisis demográfica" es el alto nivel de agregación con que se manejan las variables. Se proyectan las tendencias de los recursos globales población y tecnología y de su comparación se formulan sugerencias de política para orientar a todos los países. De esta manera se oculta una gran variedad de elementos nacionales e incluso regionales. Los múltiples factores implícitos en la tensión económica pueden variar en forma diversa y su combinación producir diversos tipos de tensiones. Más apropiado sería emprender estudios particulares, por lo menos a escala nacional, antes de fundamentar conclusiones acerca de las fuentes de la tensión económica así como de las políticas para superarla.

\section{Población y CONTAMinación}

Los niveles alarmantes de contaminación ambiental en los países desarrollados son motivo de serias inquietudes, no sólo para tales países sino también para el resto del mundo, pues en cierta medida las estructuras de producción y consumo de los países desarrollados configuraran el porvenir en los países menos desarrollados.

La literatura sobre "la crisis demográfica" en los Estados Unidos relaciona directamente la población con la contaminación. El crecimiento demográfico aumentaría la demanda total de bienes y servicios modernos, lo que se traduciría en un aumento de la contaminación ambiental, causada por la producción de dichos bienes y en parte también por su consumo.

Sin embargo, enfocar la problemática en forma exclusiva sobre la demanda impide considerar aspectos tal vez de mayor trascendencia. Se prescinde de la oferta, que comprende las decisiones en cuanto a la combinación de insumos y la tecnología que ha de utilizarse en la producción, asi como sobre las características específicas de los productos que resultarán.

En realidad los consumidores carecen de influencia directa sobre las decisiones de producción y sus consecuencias en materia de contaminación. Estas decisiones las adoptan los propietarios y gerentes (capitalistas y no capitalistas). Los consumidores, por conducto de sus decisiones de compra, ejercen una influencia indirecta y muy difusa sobre las características de los bienes que se producen y sobre la forma en que se fabrican. Su influencia es incluso menor como resultado de los medios avanzados que se utilizan para manipular las decisiones de compra del consumidor y que han surgido en los países capitalistas. Esto no niega que los consumidores puedan llegar a preferir bienes que tienen efectos contaminantes y que incluso se opongan vigorosamente a la sustitución de tales bienes por otros que fueran menos dañinos al ambiente.

Es obvio que la gran contaminación en los países desarrollados proviene de la tecnología que se utiliza. India tiene una tasa elevada de cre- 
cimiento demográfico, pero la contaminación ambiental es baja. Francia ha tenido una tasa muy baja de crecimiento demográfico, desde hace por lo menos un siglo, pero afronta graves problemas de contaminación. La diferencia reside en que Francia está industrializada e India no. Antes de imputar la contaminación a "la tecnología moderna", recordemos que la tecnología no es una entidad independiente, sino tan sólo un instrumento en manos de algunos individuos. Existen técnicas para eliminar gran parte de la contaminación que ahora experimentamos. Si esas técnicas no se aplican, la decisión no emana de "la tecnología moderna", sino de las personas que deciden sobre ella. $Y$ las personas que toman estas decisiones son predominantemente propietarios y gerentes de empresas.

Además, los costos de la contaminación así como los de la lucha contra ésta, no se distribuyen equitativamente dentro de la sociedad, de modo que, una vez más, lo que parecía constituir una conexión evidente entre la población y otro elemento, en este caso la contaminación, constituye por el contrario un problema político de gran complicación que entraña pugna de intereses entre diferentes grupos en la sociedad.

\section{Población Y URBanización}

Un aspecto característico del crecimiento económico de los países desarrollados, y actualmente también de los de menor desarrollo relativo, es el aumento de la urbanización, o sea el aumento creciente de la población que vive en zonas metropolitanas. La concentración espacial de la población, en aumento continuo, ha generado o contribuido a agudizar diversos problemas en las áreas urbanas: el empleo, la vivienda, los servicios públicos, la delincuencia, etc. En la literatura sobre "la crisis demográfica", suele establecerse un nexo entre el crecimiento demográfico y tales problemas, sugiriéndose que si la población cesara de aumentar, los problemas se simplificarían o por lo menos se atenuarían.

Nuevamente, esta descripción es demasiado simplista. La urbanización y la población han crecido juntas en la historia, en relación recíproca. La causa directa de la concentración de las actividades no agrícolas en las ciudades, es primordialmente la tecnología específica de producción y comunicaciones que se adoptó durante los siglos XIX y Xx. Esta tecnología implicaba grandes economías netas, internas y externas, por el hecho de instalarse en zonas urbanas. La tecnología moderna se ha caracterizado invariablemente por las economías de escala, que aseguran una mayor rentabilidad a las unidades económicas más grandes. La gran escala de las unidades económicas hace más provechoso concentrar la fuerza de trabajo en su vecindad. Las unidades administrativas han sido atraídas a las zonas urbanas debido a las fuertes economías que implica el hecho de hallarse cerca unas de otras y próximas a una vasta gama de servicios, lo que también conviene a las industrias. A su vez, la creación misma de centros urbanos generó más economías de localización, en un proceso dialéctico, en parte de "auto generación". Por otra parte, la transformación tecnológica en la agricultura liberó fuerza de trabajo de las zonas rurales. 
Resulta difícil advertir hasta qué punto tiene fundamento la relación entre el crecimiento demográfico y la decadencia urbana. Cabe recordar que se ha sugerido que los problemas urbanos se deben a que las ciudades rebasan su "tamaño óptimo". ${ }^{9}$ En los Estados Unidos, el crecimiento de la población suburbana en 1960-1970 fue mucho mayor que el incremento (muy lento) de la población urbana. Esto podría indicar que el tamaño de la ciudad estadounidense había rebasado el nivel óptimo, habida cuenta de la distribución del poder entre los grupos de intereses en la sociedad norteamericana. Es importante agregar esta última observación, pues nos ayuda a interpretar más acertadamente la información disponible. Si pudieran modificarse los factores estructurales asociados con las relaciones sociales de producción, la calidad de la vida en las ciudades podría alcanzar niveles humanos para la mayor parte de la población urbana en vez de limitarse a una minoría como en la actualidad.

La decadencia urbana, en vez de ser el resultado de un exceso de habitantes, constituye una expresión espacial de la distribución del ingreso y el poder en las zonas metropolitanas. En los Estados Unidos, los grupos pobres viven en el centro de la ciudad, en tanto que los grupos de ingresos medios y superiores viven en los suburbios circundantes, con núcleos dispersos de personas de altos ingresos. Las economías y deseconomías externas de las ciudades tampoco están igualmente distribuidas, la mayor parte de las deseconomías afectan a los hogares de ingresos más bajos. La estructura de los programas de renovación urbana en los Estados Unidos ha tenido una orientación clasista precisa, en el sentido de que la renovación ha consistido en la construcción subvencionada de edificios para oficinas, vivienda para personas de medianos ingresos y carreteras para acelerar la comunicación entre el centro y los suburbios y otras ciudades. Sólo una pequeña parte de los desembolsos en renovación urbana consistieron en bienes y servicios para los hogares de ingresos más bajos. El crecimiento demográfico agrava la decadencia urbana en el sentido superficial y aritmético de aumentar el número de personas de escasos recursos, pero subsiste una pregunta: ¿por qué son pobres?

\section{PoBlación y POLÍtica}

A lo largo de este ensayo ha quedado claro un aspecto importante. Es necesario ampliar el marco que se utiliza para el análisis de la interrelación entre población, economía y sociedad, de modo que se puedan incorporar factores estructurales, especialmente las relaciones sociales de producción, y la distribución del poder y de ingreso. No solamente deben incluirse estos factores en el modelo, sino que su influencia e importancia parecen esenciales. Si las ciencias sociales ignoran estos aspectos se condenan a la esterilidad y se expone deliberadamente al peligro de formular explicaciones, pronósticos y políticas, que no guardan relación con la realidad que estudian.

En la introducción se mencionó que el crecimiento demográfico ace-

9 Las definiciones sobre "tamaño óptimo" aparecen en la literatura a que nos hemos estado refiriendo sin ninguna precisión de la función objetivo o de las restricciones. 
lerado puede servir para poner de manifiesto los elementos de la estructura social que impiden a una sociedad hacer frente a los problemas del cambio. Un sistema de desigualdad y explotación que en otras circunstancias podría haber sido capaz de mantenerse con sólo pequeños disturbios, se ve seriamente amenazado por el rápido crecimiento de la población. En realidad, "la explosión demográfica" quizá sirva de impulso para modificar los factores estructurales que parecen constituir el principal coadyuvante de la tensión económica asociada al crecimiento demográfico. 